\title{
Surgical and Non-Surgical Treatment of the Lower Eyelid Fat Bulging Using Lasers and Other Energy-Based Devices
}

\author{
Sung Tae Chung \\ Nark-Kyoung Rho
}

Leaders Aesthetic Laser and Cosmetic Surgery Center, Seoul, Korea

\footnotetext{
Correspondence

Nark-Kyoung Rho

Leaders Aesthetic Laser and Cosmetic Surgery Center, 409 Dosandaero, Gangnam-gu, Seoul 06014, Korea

Tel.: +82-2-2088-7828

Fax: +82-2-2088-7829

E-mail: rhonarkahanmail.net

(C) Korean Society for Laser Medicine and Surgery

(@) This is an open access article distributed under the terms of the Creative Commons Attribution NonCommercial License (http://creativecommons.org/ licenses/by-nc/4.0) which permits unrestricted noncommercial use, distribution, and reproduction in any medium, provided the original work is properly cited.
}

Lower eyelid fat bulging is a clinically evident sign of aging. Traditionally, surgical lower blepharoplasty has been used to improve this condition but the side effects and downtime have limited its use. This review summarizes the clinical manifestations, pathophysiology, and related anatomy of lower eyelid fat bulging; describes various surgical and nonsurgical alternatives to conventional open blepharoplasty; and determines the evidence-based efficacy of these procedures. The results have been favorable for the use of surgical lasers during transconjunctival lower blepharoplasty, but most non-surgical procedures lack scientific evidence despite non-invasive energy devices being safe, tolerable, and effective tools for periorbital skin rejuvenation. The data suggests that non-invasive devices should serve as an alternative, but not an equivalent substitute for surgery.

\section{Key words}

Carbon dioxide laser; Infraorbital fat pads; Interstitial laser; Microfocused ultrasound; Radiofrequency 


\section{INTRODUCTION}

Lower eyelid fat bulging (LEFB), commonly referred to as 'baggy lower eyelids' is frequently observed in adults as a major sign of aging. ${ }^{1}$ Many individuals have an aesthetic concern with LEFB and want to improve their periorbital appearance using various interventions including surgical blepharoplasty. In 2013, people in the United States of America spent nearly $\$ 440$ million on cosmetic blepharoplasty surgery with more than 161,000 procedures performed, an increase of nearly $5.4 \%$ in procedure number from 2012. ${ }^{2}$ Although the procedure volume is increasing rapidly, surgical correction of LEFB remains a potentially challenging intervention to dermatologists because of its significant downtime and surgical complications which can lead to deformities and functional impairment. Several alternative surgical and non-surgical techniques have been described to improve LEFB; however, there remains considerable debate over the optimal standard approaches, efficacy and safety of procedures. This review aims to present current status of laser and other energybased technologies offering improvement of LEFB and the scientific evidence underlying them. Pathophysiology and the relevant anatomy of LEFB is also presented for better understanding of procedures.

\section{PATHOPHYSIOLOGY AND ANATOMY}

Various anatomic and physiologic changes are related to periorbital changes in Asian patients. Understanding of the complex factors that contribute to periorbital changes and LEFB during aging process may allow for adequate and customized correction for each patient and help in prevention of complications.

\section{Pathophysiology and clinical manifestations}

Although the volume ratio of inferior orbital fat to total orbital fat increases with age, ${ }^{3}$ the aging-related weakening of the orbital septa is thought to be an primary cause of LEFB. ${ }^{4}$ It results in the progressive herniation of one or more of the three infraorbital fat pads (Fig. 1); thus, the conventional blepharoplasty usually involves removing herniated fat through the eyelid skin or conjunctiva. ${ }^{1}$ Using data of orbital and facial computed tomography from 167 Chinese patients, Chen et al. ${ }^{5}$ evaluated curvature and protrusion of lower eyelid fat and its changes in different age groups and concluded that although the amount of lower eyelid fat varies greatly between individuals, the process of lower eyelid fat herniation does occur regardless of the size of the fat pads. They also found that

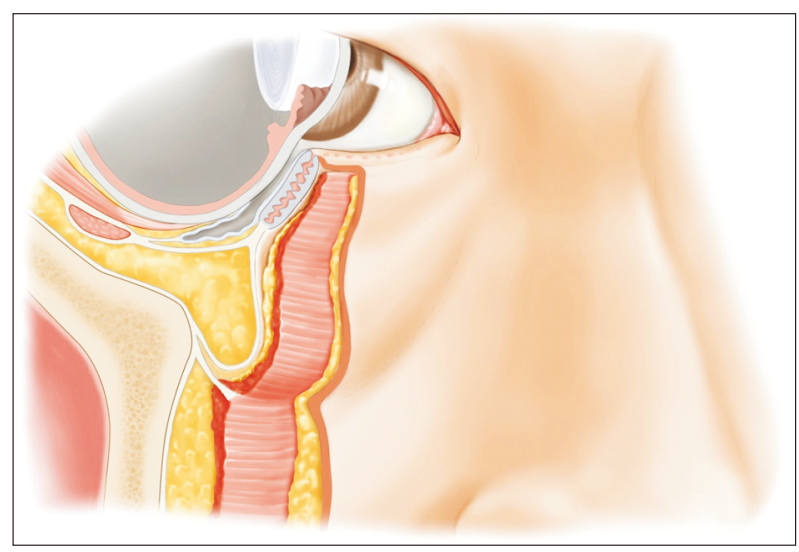

Fig. 1. Lower eyelid fat bulging and its anatomical correlation.

the degree of LEFB retains a steady incline after the third decade of life and slows at approximately age 70.

Jo et al. ${ }^{1}$ analyzed frontal-view photographs of the faces of the 1,034 Korean patients who sought for correction of LEFB. Based on the clinical pattern, they classified the LEFB into medial (M), medial and central (MC), and medial to lateral (MCL) types. They found out that the most common types were MCL type in the men and MC type in the women, respectively. The $M$ and $M C$ types were common in the young subjects, whereas the MCL type was more common in the old subjects. The severity of the LEFB showed positive correlation with age, a higher mean value in the men, and a trend toward higher values in the order of M, MC, and MCL types. ${ }^{1}$

Goldberg et al. ${ }^{6}$ and colleagues analyzed the histories and photographs of 114 patients whose motivation for aesthetic consultation was lower eyelid contour and identified 6 categories of anatomic basis (cheek descent and hollow tear trough, prolapse of orbital fat, skin laxity and sun damage, eyelid edema, orbicularis muscle hyperactivity, and triangular cheek festoon). According to their results, LEFBs do not have a single anatomic basis and the authors concluded that for different anatomic problems, different treatments should be recommended.

\section{Surgical anatomy}

The orbital septum of the lower eyelid arises as a fibrous extension of the arcus marginalis. The orbital septum fuses with the lower eyelid retractors below the tarsal plate (Fig. 2). In Caucasians, this conjoined area begins 3-4 mm lower than it does in Asians. As a result of these anatomic differences, the length of unreinforced septum is about $12.3 \mathrm{~mm}$ in Asians and only $9.3 \mathrm{~mm}$ in Caucasians. Attenuation of the inferior orbital septum thereby allows the lower eyelid fat pads to easily bulge in 


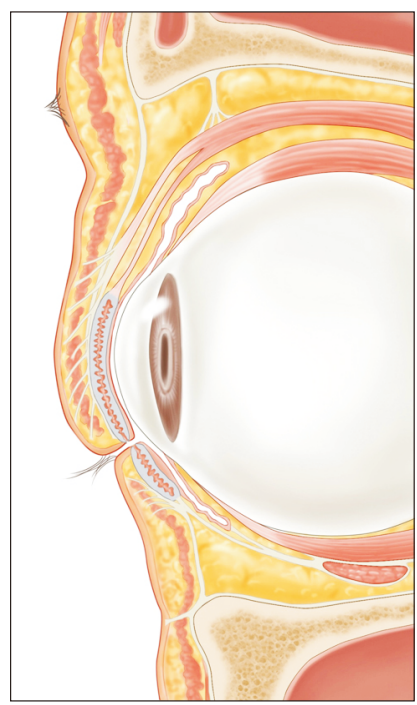

Fig. 2. Orbital septum and other structures contributing to the lower eyelid fat bulging.

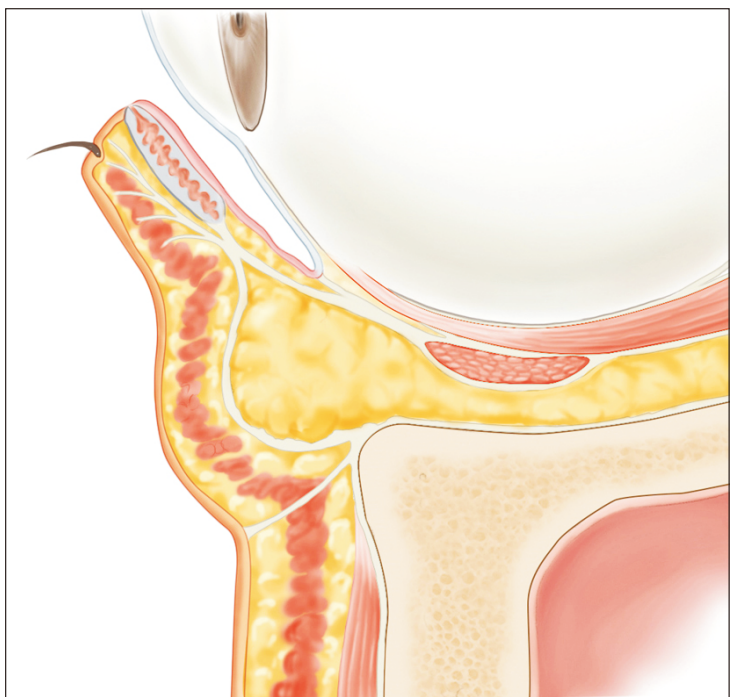

Fig. 3. Attenuation of the inferior orbital septum and the resultant bulging of lower eyelid fat pads.

\section{Asians (Fig. 3). ${ }^{7}$}

There are three clinically apparent fat pads in the lower eyelid: the medial, central, and lateral fat pads. In many cases, the larger medial fat pad is often subdivided into two smaller collections (medial and central) by a septum in the region of the inferior oblique muscle origin, while its arcuate expansion separates the central and lateral fat pads (Fig. 4). Anatomic variations do exist where only two compartments or a non-compartmentalized single fat pad is found. A pretarsal fat pad has also been described and is located at the lateral half of the tarsal plate, just

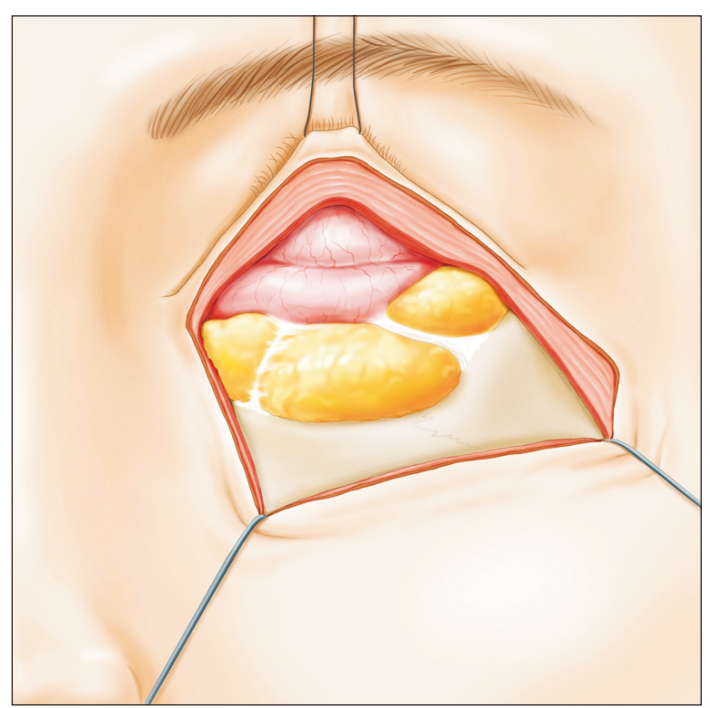

Fig. 4. Three fat pads in the lower eyelid.

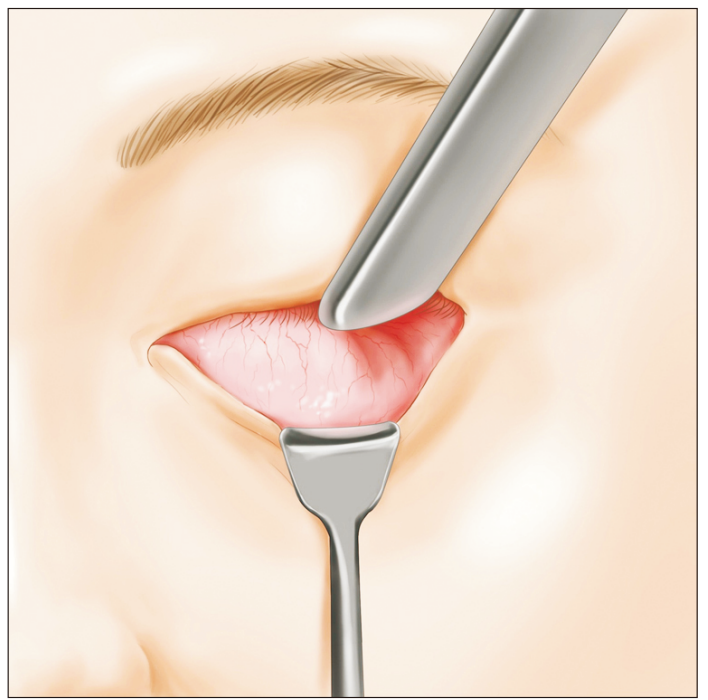

Fig. 5. Vascular arcades of the lower eyelid.

superior to the lateral fat pad. ${ }^{8}$ This pretarsal fat can contribute to the visible bulk of the eyelid below the eyelashes.

The extensive vascularity of the eyelids promotes healing and helps defend against infection. The arterial supply of the eyelids comes from 2 main sources: (1) the internal carotid artery by way of the ophthalmic artery and its branches (supraorbital and lacrimal) and (2) the external carotid artery by way of the arteries of the face langular and temporal). Collateral circulation between these two systems is extensive, anastomosing throughout the upper and lower eyelids and forming the marginal and peripheral arcades (Fig. 5). The lower eyelid often has only 1 
arterial arcade, located at the inferior tarsal border. ${ }^{9}$

\section{SURGICAL PROCEDURES}

\section{Carbon dioxide laser-assisted transconjunctival lower blepharoplasty}

The transconjunctival approach to lower blepharoplasty was first described by Bourquet in 1923 and, more recently, a technique that combines a transconjunctival approach to resect fat with skin excision using either a skin flap or pinch excision has been described. ${ }^{10}$ This inside/ outside approach spares the intervening orbicularis oculi muscle and avoids long-term sequelae of denervation atrophy seen with skin-muscle flaps. ${ }^{11}$ The transconjunctival approach may look easier than the transcutaneous technique, however, it requires precise anatomical knowledge of the internal region. The main risk point is difficulty in bleeding control. ${ }^{12}$ Meticulous hemostasis is critical during the entire procedure. The blood vessels associated with each fat compartment must be cauterized. In the late 1980s, the introduction of carbon dioxide $\left(\mathrm{CO}_{2}\right)$ lasers in the aesthetic surgery has permitted the development of new approaches toward the LEFB. The high absorption of $\mathrm{CO}_{2}$ laser by tissue water gives surgeons the opportunity to perform excisional and incisional procedures with simultaneous homeostasis. ${ }^{13}$ Currently, many surgeons use $\mathrm{CO}_{2}$ lasers both for the standard lower incisional blepharoplasty through a subciliary incision and for the transconjunctival approach where laser resurfacing is performed right after the surgical procedure. ${ }^{13}$

For the transconjunctival approach, an injection of $2 \%$

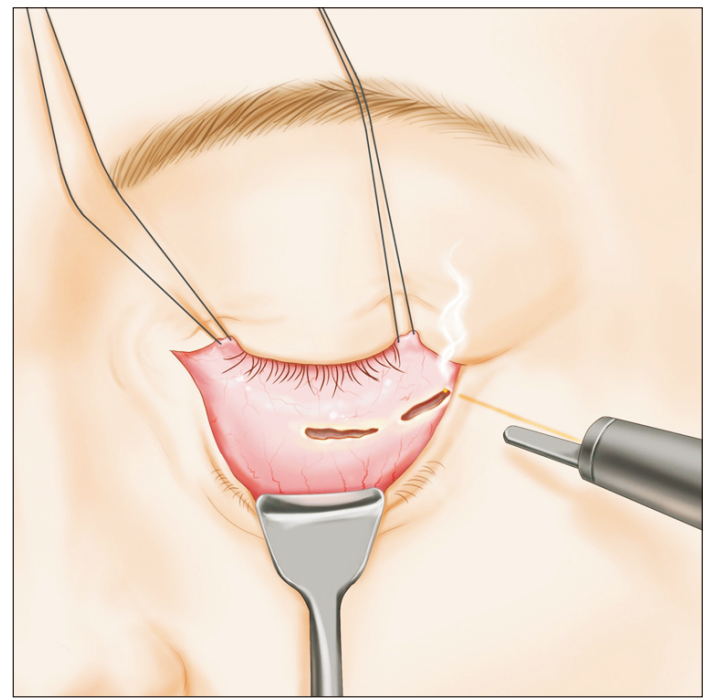

Fig. 6. Conjunctival incision using a carbon dioxide $\left(\mathrm{CO}_{2}\right)$ laser to expose the inferior orbital fat pads. lidocaine with 1:100,000 epinephrine is made in the lower eyelid through the inferior fornix. A Desmarres retractor is used to retract the lower eyelid away from the globe and expose the inferior fornix. Then, using the continuous wave mode with 4-7 watts of power, the lower eyelid conjunctiva is incised by $\mathrm{CO}_{2}$ laser to expose the inferior orbital fat pads (Fig. 6). There are two types of conjunctival incision: preseptal and retroseptal (Fig. 7). ${ }^{14}$ The preseptal dissection plane is performed through a conjunctival incision, $2 \mathrm{~mm}$ under the inferior edge of the tarsus. To reach the fat, the septum must be incised following separation of the septum and the orbicularis oculi muscle. This technique is used to minimize bleeding and fat extrusion into the operating field. Many septal punctures are necessary in this technique to reach all the fatty chambers, as in the classic external approach. The retroseptal dissection plane is performed through a lower conjunctival incision, 3 to $5 \mathrm{~mm}$ under the inferior edge of the tarsus, avoiding the inferior conjunctival fornix. This approach provides a direct access to the inferior orbital fat without wounding the septum. In either approach, care should be taken to minimize bleeding from the conjunctival vascular arcades.

A small Desmarres retractor is then inserted to retract the lower eyelid. This burnished retractor should diffuse, rather than reflect, any laser light that hits it. Next, the lower eyelid retractors are pulled superiorly out of the incision, which brings the fat up with it. Fat is teased from the central compartment with forceps and the arcuate expansion is exposed. This is incised to allow the central and lateral fat pads to become continuous. The central

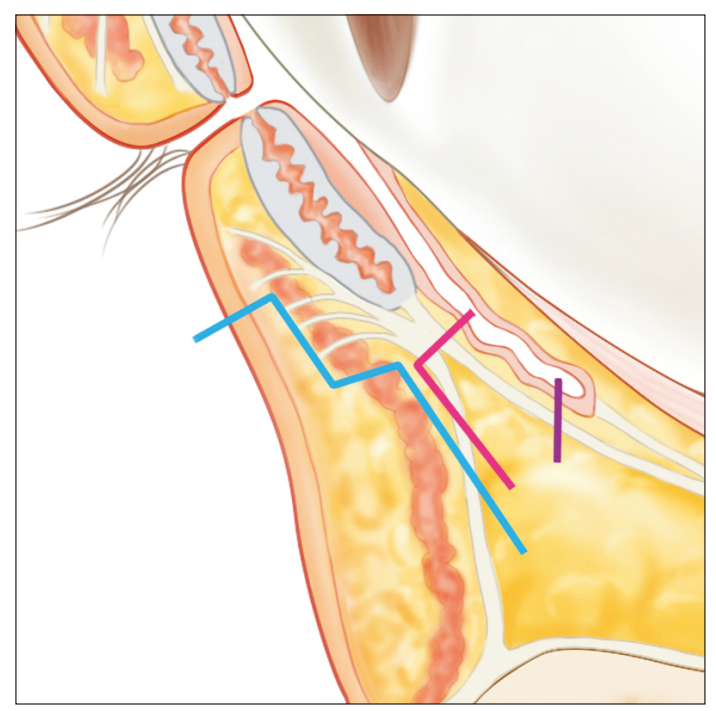

Fig. 7. Various approaches to lower eyelid incision: (blue) subciliary; (red) transconjunctival, preseptal; (purple) transconjunctival, retroseptal. 
and lateral fat pads are generally removed first. The fat is clamped and excised directly by laser (Fig. 8). By using a $\mathrm{CO}_{2}$ laser, cautery is provided simultaneously during excision. The medial fat compartment is the most difficult to locate and is bounded laterally by the inferior oblique muscle. Extreme care should be taken in this area. The inferior oblique muscle can usually be identified once the fat is removed. If this fat pad is not very prominent, dissection is not recommended. Volumes of excised fat pads are checked (Fig. 9) and the degree of residual fat bulging is evaluated. The endpoint is when the fat is removed down to and flushed with the inferior orbital rim. Generally, no sutures are placed unless the incision is extensive. Antibiotic ophthalmic ointment and ice compresses are used postoperatively.

Postoperative retro-orbital hemorrhage is the most feared complication following lower blepharoplasty. ${ }^{12}$ It can be accompanied with pain and decreased visual acuity with pupillary defect. Edema of the orbicularis muscle

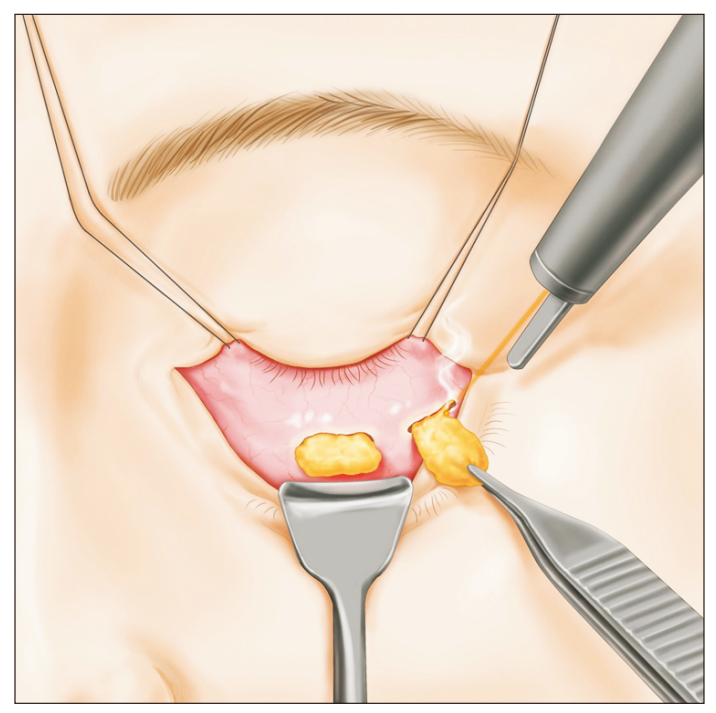

Fig. 8. Excision of the fat pads using a $\mathrm{CO}_{2}$ laser.

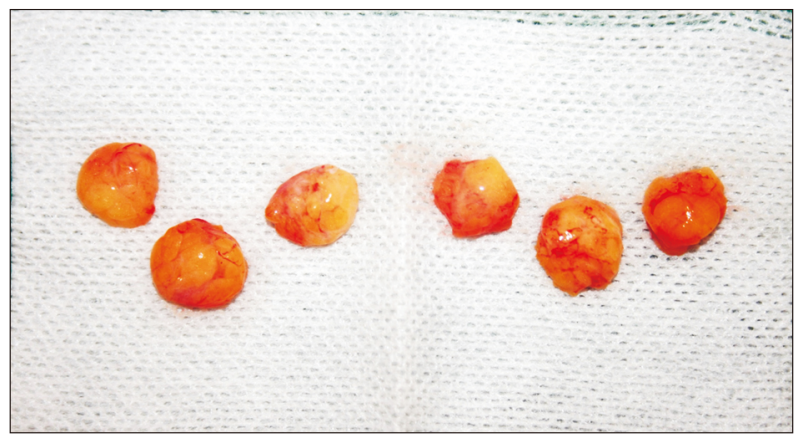

Fig. 9. Excised fat pads. due to hemorrhage can affect visual acuity. Other complications include infection, scleral show, conjunctivitis, corneal ulcer, diplopia, amaurosis, and damage of both the inferior oblique muscle and the orbicularis oculi muscle. According to the results of Rancati et al., ${ }^{12}$ insufficient lipectomy is more common in the transconjunctival group than in the transcutaneous blepharoplasty group. On the other hand, overresection of the fat pads frequently results in the hollowing of the infraorbital region along with the worsening of the lower eyelid wrinkles. Final clinical outcome is evaluated at least two months after the procedure (Fig. 10). Laser or radiofrequency (RF) resurfacing is recommended postoperatively to improve postoperative worsening of fine wrinkles.

\section{Interstitial lasers}

Laser lipolysis with pulsed lasers, also called interstitial lasers, has been introduced and continues to improve as clinicians search for faster, more effective and safer ways to reduce localized fat deposits. This technology has been demonstrated to result in degenerated fat cell membrane, vaporization, liquefaction, carbonization, and heatcoagulated collagen fibers ${ }^{15}$ while causing less downtime and side effects when compared to traditional liposuction. Although not commonly performed, laser-assisted lipolysis has been used as an alternative to traditional surgical procedures for LEFB. ${ }^{16}$ A typical example of the interstitial laser system is a neodymium-doped yttrium-aluminumgarnet (Nd:YAG) laser operating in the infrared portion of the spectrum $(1,064 \mathrm{~nm})$, with the photomechanical force and energy delivered via fiberoptics through a 1-mm cannula. Zhang et al. ${ }^{16}$ retrospectively reviewed 128 cases of transconjunctival laser-assisted lipolysis procedures. The procedure was basically similar to traditional transcon-

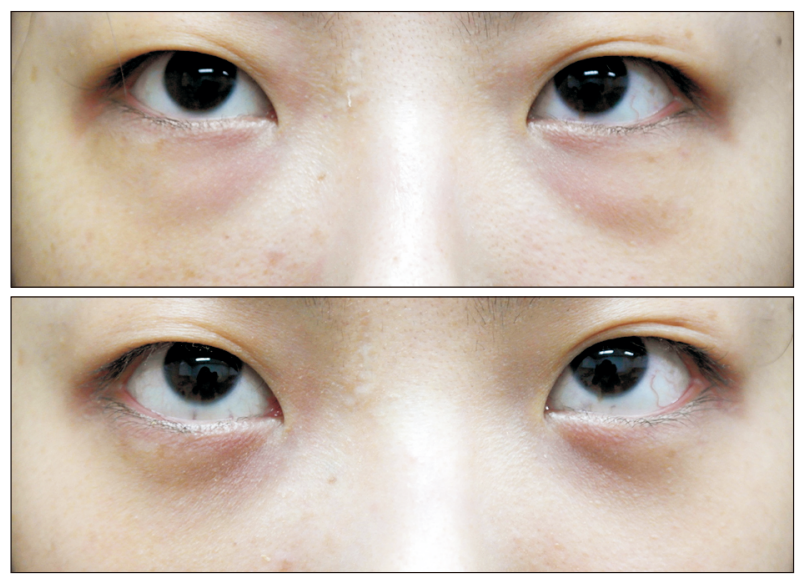

Fig. 10. Clinical photography comparing before and 2 months after $\mathrm{CO}_{2}$ laser-asssisted transconjunctival lower bleopharoplasty. 
junctival lower blepharoplasty. Under local anesthetics injection, a small hole was made through the palpebral conjunctiva into the septum at a mean 4-8 $\mathrm{mm}$ inferior to the edge of the inferior tarsus plate. The cannula with 1,064-nm Nd:YAG laser fiber was introduced into the septum slowly through the hole. Standard laser parameters were $20 \mathrm{~Hz}$ frequency and an average 450 joules of energy delivered at $20 \mathrm{~Hz}$ and $3 \mathrm{~W}$. The adipocytes were broken up under the accumulated photomechanical effect and the operator was able to see the liquified fat coming out through the hole. Gauze soaked in sterile ice water was used to cover the lower eyelid during the entire procedure to keep the skin cold. Standard post-blepharoplasty cares included rest, anti-inflammatory and antibiotic eye drops, lubricant ophthalmic ointment and ice pads. Apart from the edema and chemosis, which resolved spontaneously within days, no serious complications, such as damage to the vision, ocular functional impairment, skin burns and scarring, were found. Clinical efficacy was comparable to that of transconjunctival lower blepharoplasty.

A complication report of a 54-year-old female who underwent laser lipolysis blepharoplasty highlights the danger of the overheating. ${ }^{17}$ The laser system used was an interstitial $980-\mathrm{nm}$ diode laser and the procedure was performed with transcutaneous approach. A fullthickness dermal burn developed after the procedure. It is recommended that during laser-assisted lipolysis of

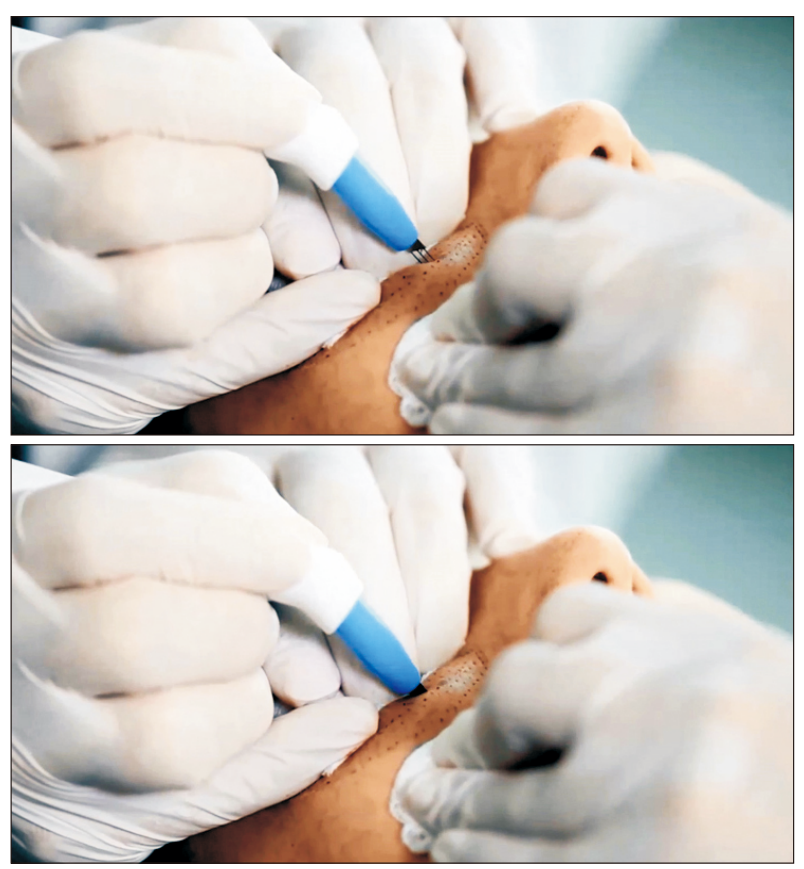

Fig. 11. The use of insulated microneedle-radiofrequency system for the improvement of lower eyelid fat pad bulging. the lower eyelid the surface temperature be maintained below $42^{\circ} \mathrm{C}$ when treating superficially to avoid burns and scarring. ${ }^{18}$

\section{MINIMALLY INVASIVE PROCEDURES}

\section{Fractionated microneedle RF}

Fractional microneedle RF was developed with the same conceptual framework as fractional lasers, which is to provide focal, high-energy treatment zones within intact skin for the purpose of reduced downtime and risk. This technology has been applied with a variety of applicator geometries, however, its use for the LEFB has not been reported in the literature. ${ }^{19,20}$ The superficial placement of needle tip and short pulse duration of conventional microneedle-type RF devices make this technology not suitable for volume reduction of inferior orbital fat. In the authors' opinion, fractionated microneedle RF can be better used in combination with transconjunctival lower blepharoplasty because this technology has been shown to tighten lower eyelid skin with less downtime and minimal side effects. ${ }^{19,20}$ A more aggressive type of microneedle RF system such as Profound ${ }^{\mathrm{TM}}$ (SyneronCandela, Wayland, MA, USA) ${ }^{21}$ or AGNES ${ }^{\text {TM }}$ (Gowoonsesang Cosmetics, Seoul, Korea ${ }^{22}$ can be an option for the treatment of LEFB. Under local anesthetics infiltration, the microelectrodes are introduced from the lower eyelid skin into the bulging infraorbital fat pads where the impedance is significantly higher than the reticular dermis and RF pulses with longer duration (several seconds) are irradiated to achieve adipocyte destruction and septal

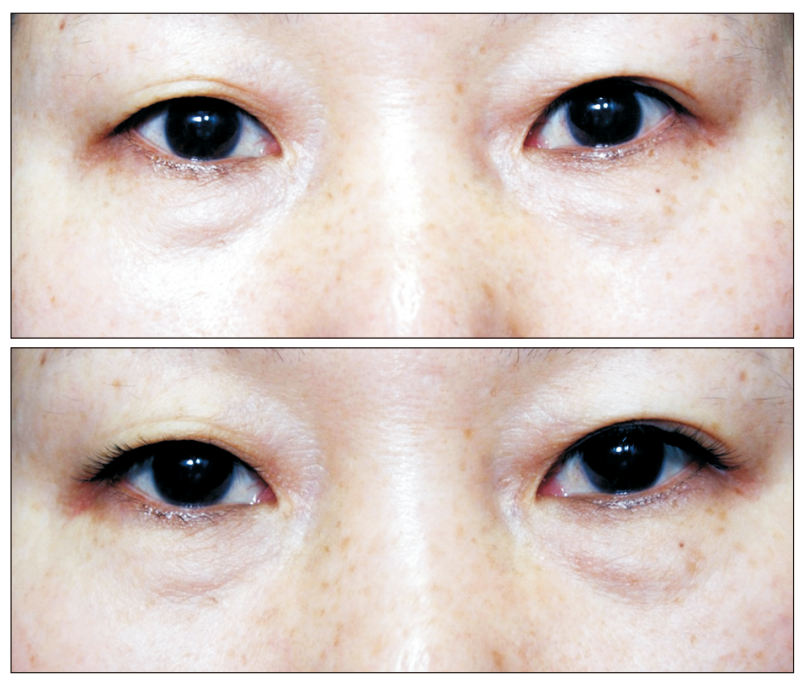

Fig. 12. Mild improvement of the lower eyelid fat bulging after insulated microneedle-radiofrequency treatment. 
tightening (Fig. 11, 12). In a recent 24-week prospective trial involving 24 Korean patients, the efficacy of safety of microinsulated needle RF system for the treatment of LEFB has been presented. ${ }^{23}$

\section{NON-INVASIVE PROCEDURES}

\section{Non-ablative RF}

The demand for noninvasive methods of facial rejuvenation has experienced exponential growth over the last decade. Non-invasive and non-ablative RF facial treatments are optimal for those wishing to avoid recovery time. ${ }^{24}$ Various non-ablative monopolar and bipolar RF devices are being used to tighten facial skin. According to Biesman et al. ${ }^{25}$ who evaluated the efficacy of a small (0.25 $\mathrm{cm}^{2}$ ) monopolar RF treatment tip (Thermage ${ }^{\mathrm{TM}}$ Eye; Solta Medical, Hayward, CA, USA) for aesthetic rejuvenation of the eyelids, upper eyelid tightening and reduction of hooding was noted in 88 and $86 \%$ of subjects, respectively and lower eyelid tightening was noted in $71-74 \%$ of subjects. There were no serious adverse sequelae. Although the clinical efficacy of this procedure is high in terms of eyelid skin tightening, the authors think that non-ablative RF devices do not provide infraorbital fat volume reduction. A non-ablative RF eyelid skin tightening is a good treatment option when used in combination with surgical or nonsurgical procedures for the LEFB.

\section{Microfocused ultrasound}

Microfocused ultrasound (MFU), also known as high intensity focused ultrasound, generates tissue heating, which can be used for facial skin tightening as well as other areas of skin laxity. Unlike radiofrequency energy, MFU can penetrate deep into tissue without heating the superficial skin structures, allowing for higher treatment temperatures. ${ }^{26}$ The Ulthera ${ }^{\mathrm{TM}}$ system (Ulthera, Inc.,

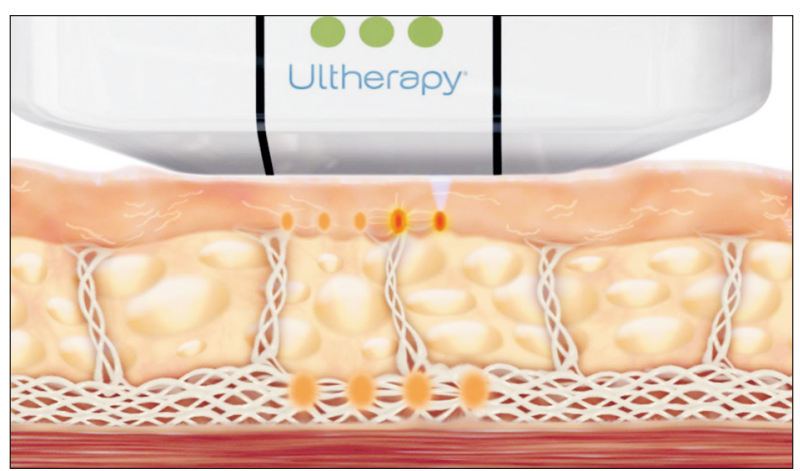

Fig. 13. A schematic illustration describing the use of microfocused ultrasound for skin rejuvenation.
Mesa, AZ, USA) has refined MFU technology using transducer handpieces uniquely capable of imaging mode and treatment mode. ${ }^{26}$ Ulthera ${ }^{\mathrm{TM}}$ transducers produce fractional microcoagulation zones at 1.5, 3.0 and $4.5 \mathrm{~mm}$ focal depths from the skin surface (Fig. 13). This system has been widely used for the tightening and lifting of face and neck skin, ${ }^{27}$ including infraorbital areas. Suh et al. ${ }^{28}$ assessed the safety and efficacy of Ulthera ${ }^{\mathrm{TM}}$ system for skin tightening in 15 Korean patients with infraorbital laxity. In their study, a $7.0 \mathrm{MHz}, 3.0 \mathrm{~mm}$ focal depth transducer was used to deliver a single pass over the thin infraorbital area to generate micro-thermal coagulation zones. The spacing between the pulses was set to approximately 3.0-5.0 $\mathrm{mm}$. The energy per ultrasound pulse ranged from 0.25 to $0.35 \mathrm{~J}$. The procedure was performed under topical anesthetic cream application. Six months after a single treatment session, all patients experienced both subjective and objective improvement. Post treatment biopsy specimens showed regenerated and increased collagen and elastic fibers in the reticular dermis. No serious, permanent, or delayed side effects were noted up to 6 months post treatment. The researchers concluded that this procedure can be effective and safe in the treatment of decreased laxity of the lower eyelids. What is interesting in their study is that the before and after photographs of the patients clearly show the decrease of LEFB alongside with the improvement of lower eyelid wrinkles and sagging. The $3.0 \mathrm{~mm}$ depth of the MFU-induced focal coagulation zones anatomically match the superficial parts of infraorbital fat pads. Given that MFU has been shown to produce controlled rise in temperature up to $70^{\circ} \mathrm{C}$ at the focal zone and disrupts and destroy fat cells, while raising the temperature of collagen fibers causes them to thicken and contract, ${ }^{29}$ MFU can be a future non-invasive option for the treatment of LEFB.

\section{CONCLUSION}

Lower blepharoplasty has been referred to as one of the more difficult procedures in the practice of aesthetic surgery due to the complications range from a subtle change in eye shape to frank ectropion with corneal ulceration. ${ }^{30}$ Transconjunctival blepharoplasty surgery can be benefited from or modified by the use of surgical lasers, interstitial lasers, or ablative RF devices. Although the clinical efficacy is not comparable to surgical interventions, several non-invasive modalities can be used to improve LEFB phenomenon without significant downtime and complications. To get consistent and reproducible results, these non-invasive procedures needs more sci- 
entific data. Development of new invasive or non-invasive energy-based devices specifically targeting LEFB will bring new opportunities in the field of aesthetic medicine and surgery.

\section{ACKNOWLEDGEMENTS}

The authors would like to deeply thank Ms. Suhyun Chae, MFA, Devision of Biomedical Art, Department of Fine Art, Incheon Catholic University Graduate School, Incheon, Korea for the beautiful and informative illustrations she drew to our exact requirements.

\section{REFERENCES}

1. Jo SJ, Kim HS, Park JT, Kim BR, Youn SW. Assessment of ageand sex-related changes in baggy lower eyelids using a novel objective image analysis method: orbital gray scale analysis. J Cosmet Dermatol. In press 2017.

2. Kossler AL, Peng GL, Yoo DB, Azizzadeh B, Massry GG. Current trends in upper and lower eyelid blepharoplasty among American society of ophthalmic plastic and reconstructive surgery members. Ophthal Plast Reconstr Surg. In press 2017.

3. Lee JM, Lee H, Park M, Lee TE, Lee YH, Baek S. The volumetric change of orbital fat with age in Asians. Ann Plast Surg 2011;66:192-5.

4. Hamra ST. Arcus marginalis release and orbital fat preservation in midface rejuvenation. Plast Reconstr Surg 1995;96:35462.

5. Chen YS, Tsai TH, Wu ML, Chang KC, Lin TW. Evaluation of agerelated intraorbital fat herniation through computed tomography. Plast Reconstr Surg 2008;122:1191-8.

6. Goldberg RA, McCann JD, Fiaschetti D, Ben Simon GJ. What causes eyelid bags? Analysis of 114 consecutive patients. Plast Reconstr Surg 2005;115:1395-402.

7. Kakizaki H, Jinsong Z, Zako M, Nakano T, Asamoto K, Miyaishi O, et al. Microscopic anatomy of Asian lower eyelids. Ophthal Plast Reconstr Surg 2006;22:430-3.

8. Hwang K, Kim DJ, Chung RS. Pretarsal fat compartment in the lower eyelid. Clin Anat 2001;14:179-83.

9. Tucker SM, Linberg JV. Vascular anatomy of the eyelids. Ophthalmology 1994;101:1118-21.

10. Zarem HA, Resnick JI. Expanded applications for transconjunctival lower lid blepharoplasty. Plast Reconstr Surg 1991;88:21520.

11. Hidalgo DA. An integrated approach to lower blepharoplasty. Plast Reconstr Surg 2011;127:386-95.

12. Rancati A, Jacovella P, Zampieri AE, Dorr J, Daniele M, Liedtke $S$, et al. Lower blepharoplasty review, transconjunctival vs. transcutaneous approach. Mod Plast Surg 2015;5:1-8.
13. Griffin RY, Sarici A, Ozkan S. Treatment of the lower eyelid with the $\mathrm{CO} 2$ laser: transconjunctival or transcutaneous approach? Orbit 2007;26:23-8.

14. Korchia D, Braccini F, Paris J, Thomassin J. Transconjunctival approach in lower eyelid blepharoplasty. Can J Plast Surg 2003;11:166-70.

15. Ichikawa K, Miyasaka M, Tanaka R, Tanino R, Mizukami K, Wakaki M. Histologic evaluation of the pulsed Nd:YAG laser for laser lipolysis. Lasers Surg Med 2005;36:43-6.

16. Zhang $Y$, Zhang $L$, Sun $X$, Zhu $Y$, Jin R, Sun B, et al. Evaluation of lower blepharoplasty treated with the SmartLipo 1064-nm system and its clinical implications: a retrospective review. J Cosmet Laser Ther 2016;18:376-80.

17. Yu D, Biesman B, Khan JA. Bilateral eyelid dermal burn from subcutaneous diode laser lipolysis blepharoplasty. Lasers Surg Med 2009;41:609-11.

18. DiBernardo BE, Reyes J, Chen B. Evaluation of tissue thermal effects from 1064/1320-nm laser-assisted lipolysis and its clinical implications. J Cosmet Laser Ther 2009;11:62-9.

19. Kim JK, Roh MR, Park GH, Kim YJ, Jeon IK, Chang SE. Fractionated microneedle radiofrequency for the treatment of periorbital wrinkles. J Dermatol 2013;40:172-6.

20. Lee SJ, Kim JI, Yang YJ, Nam JH, Kim WS. Treatment of periorbital wrinkles with a novel fractional radiofrequency microneedle system in dark-skinned patients. Dermatol Surg 2015;41:615-22.

21. Alexiades-Armenakas M, Rosenberg D, Renton B, Dover J, Arndt K. Blinded, randomized, quantitative grading comparison of minimally invasive, fractional radiofrequency and surgical face-lift to treat skin laxity. Arch Dermatol 2010;146:396-405.

22. No YA, Kim BJ, Kim MN, Hong CK. The clinical experience and efficacy of radiofrequency device for wrinkle treatment. Lasers Med Sci 2017;32:1449-50.

23. Huh $\mathrm{CH}$. Nonsurgical treatment of eye bag using microinsulated needle radiofrequency (RF). Presented at ASDS Annual Conference, Chicago, 2017

24. Beasley KL, Weiss RA. Radiofrequency in cosmetic dermatology. Dermatol Clin 2014;32:79-90.

25. Biesman BS, Baker SS, Carruthers J, Silva HL, Holloman EL. Monopolar radiofrequency treatment of human eyelids: a prospective, multicenter, efficacy trial. Lasers Surg Med 2006;38:890-8.

26. Gutowski KA. Microfocused ultrasound for skin tightening. Clin Plast Surg 2016:43:577-82.

27. Carruthers J, Burgess C, Day D, Fabi SG, Goldie K, Kerscher $M$, et al. Consensus recommendations for combined aesthetic interventions in the face using botulinum toxin, fillers, and energy-based devices. Dermatol Surg 2016;42:586-97.

28. Suh DH, Oh YJ, Lee SJ, Rho JH, Song KY, Kim NI, et al. A intense-focused ultrasound tightening for the treatment of in- 
fraorbital laxity. J Cosmet Laser Ther 2012;14:290-5.

29. Gadsden E, Aguilar MT, Smoller BR, Jewell ML. Evaluation of a novel high-intensity focused ultrasound device for ablating subcutaneous adipose tissue for noninvasive body contouring: safety studies in human volunteers. Aesthet Surg J
2011;31:401-10.

30. Codner MA, Wolfli JN, Anzarut A. Primary transcutaneous lower blepharoplasty with routine lateral canthal support: a comprehensive 10-year review. Plast Reconstr Surg 2008;121:241-50. 